\title{
Association of Potentially Inappropriate Medications With All-Cause Mortality in the Elderly Acute Decompensated Heart Failure Patients: Importance of Nonsteroidal Anti-Inflammatory Drug Prescription
}

\author{
Tomiko Sunaga ${ }^{\mathrm{a}, \mathrm{b}, \mathrm{e}}$, Azusa Yokoyama ${ }^{\mathrm{b}}$, Shoko Nakamura ${ }^{\mathrm{a}}$, Nagisa Miyamoto ${ }^{\mathrm{b}}$, Saki Watanabec, \\ Miki Tsujiuchi ${ }^{\mathrm{d}}$, Sakura Nagumo ${ }^{\mathrm{d}}$, Ayaka Nogid ${ }^{\mathrm{d}}$, Hideyuki Maezawa ${ }^{\mathrm{d}}$, Takuya Mizukamid, \\ Mio Ebato ${ }^{d}$, Hiroshi Suzuki ${ }^{d}$, Akihiro Nakamura ${ }^{\mathrm{c}}$, Toru Watanabe ${ }^{\mathrm{a}, \mathrm{b}}$, Tadanori Sasaki ${ }^{\mathrm{a}}$
}

\begin{abstract}
Background: Acute decompensated heart failure (ADHF) is the most common cause of readmissions in the hospital. ADHF patients are associated with polypharmacy. It is a common problem among elderly patients due to frequently occurring multiple morbidities and is associated with the use of potentially inappropriate medications (PIMs). The aim of this study was to examine the association between PIMs and all-cause mortality in elderly ADHF patients.
\end{abstract}

Methods: This retrospective study included ADHF patients who were admitted to the Showa University Fujigaoka Hospital between January 2015 and August 2016. We investigated the proportion of patients taking at least one PIM at admission and the characteristics of patients at admission. PIMs were defined based on the Screening Tool of Older People's potentially inappropriate Prescriptions (STOPP). Multiple Cox regression analysis was performed to examine the association between PIM use and all-cause mortality.

Results: A total of 193 elderly patients (median age 81 years, interquartile range (IQR) 65 - 99 years) were included in the study. Allcause death occurred in 30 patients. The median number of medications at admission was 7 (IQR 0 - 18). The number of medications (greater than or equal to six) at admission was associated with mortal-

Manuscript submitted April 19, 2020, accepted May 7, 2020

Published online June 3, 2020

aDepartment of Hospital Pharmaceutics, School of Pharmacy, Showa University, Tokyo, Japan

bDepartment of Pharmacy, Showa University Fujigaoka Hospital, Kanagawa, Japan

cDivision of Pharmaceutics, Department of Pharmacology, Toxicology and Therapeutics, School of Pharmacy, Showa University, Tokyo, Japan

${ }^{\mathrm{d}}$ Division of Cardiology, Department of Internal Medicine, Showa University Fujigaoka Hospital, Kanagawa, Japan

${ }^{e}$ Corresponding Author: Tomiko Sunaga, Department of Pharmacy, Showa University Fujigaoka Hospital, 1-30 Fujigaoka, Aoba, Yokohama, Kanagawa 227-8501, Japan. Email: tomiko-s@cmed.showa-u.ac.jp

doi: https://doi.org/10.14740/cr1078 ity. Multivariate Cox regression analysis revealed that systolic blood pressure (SBP) $<100 \mathrm{~mm} \mathrm{Hg}$ at admission, chronic obstructive pulmonary disease (COPD), and use of non-steroidal anti-inflammatory drugs (NSAIDs) at admission were independent predictors for allcause mortality.

Conclusions: The medical staff should attempt to stop unnecessary medications that are prone to be inappropriate prescribing. In particular, prescription of NSAIDs should be carefully assessed and monitored.

Keywords: Elderly heart failure; Potentially inappropriate medications; Prognosis; Non-steroidal anti-inflammatory drugs; STOPP criteria

\section{Introduction}

In Japan, the number of patients with heart failure (HF) is presumed to be more than one million people, and the incidence of HF in elderly people has been increasing with the aging society [1]. In particular, it is thought that the "heart failure pandemic" is predicted to occur and there will be an increase in HF patients in 2030, which is expected to become a social problem. In developed countries, $\mathrm{HF}$ is the most common cause of readmissions in hospitals. Similarly, in Japan, repeated hospital admission due to worsening HF is common and a serious problem. Kaneko et al reported that the cumulative rate of readmission was $17.5 \%$ at 1 year, $21.4 \%$ at 2 years, and $25.5 \%$ at 3 years [2].

Recently, polypharmacy has emerged as a common problem among elderly patients due to the high prevalence of multiple morbidities $[3,4]$. It is associated with the use of potentially inappropriate medications (PIMs) $[5,6]$. In addition, it has been demonstrated that the higher risk of use of any PIMs at discharge is independently associated with female sex and the increasing number of medications at admission [7]. Benzodiazepines (BZPs), non-BZP hypnotics, and proton pump inhibitors (PPIs), in particular, have been reported to be the most 
widely used PIMs at admission and discharge.

HF patients are associated with polypharmacy $[8,9]$ and are known to have multiple morbidities [10]. It has been suggested that higher frequency of multiple morbidities in HF patients may lead to increased polypharmacy with consequential increases in drug-drug or drug-disease interactions and drugrelated adverse effects $[9,11]$. Elderly adults in particular who have multiple manifestations of cardiovascular diseases (e.g. hypertension, coronary artery disease, HF, atrial fibrillation/ flutter) are prescribed medications that need to be taken for a long period due to the chronic nature of their diseases.

To identify PIMs in elderly patients, various tools have been developed, including the Beers Criteria [12] and the Screening Tool of Older People's potentially inappropriate Prescriptions (STOPP) [13]. In 2015, the Japan Gerontological Society published an update to the "Guidelines for medical treatment and its safety in the elderly" as a screening tool for PIMs [14]. Although these tools reduce the prescription of PIMs, there is currently no disease-specific list available to assess PIM use for HF patients. In the St Vincent's PIMs study, it has been shown that the Consensus Potentially Inappropriate Medicines in Heart Failure (PIMHF) list provided the first HFspecific medicine review tool [15]. The PIMHF list consisted of 11 items including non-steroidal anti-inflammatory drugs (NSAIDs), thiazolidinediones, metformin, cyclo-oxygenase (COX)-2 inhibitors, and pregabalin.

However, currently, there is no robust evidence to support the application of medicine review using validated tools in practice [15] and only few studies have examined the association between PIMHF and clinical outcome. Furthermore, in Japan, because insurance medical treatment differs from that in other countries, it is difficult to compare the results in PIMHF. Therefore, the present study aimed to examine the association between the clinical factors associated with PIMHF use at admission and all-cause mortality in acute decompensated heart failure (ADHF) patients.

\section{Materials and Methods}

\section{Participants}

This retrospective study included ADHF patients who were admitted to the Showa University Fujigaoka Hospital between January 2015 and August 2016. The inclusion criterion was as participants aged $\geq 65$ years with ADHF. Exclusion criteria were participants aged $<65$ years or whose data were missing. We classified the participants with and without PIMs and analyzed their demographic characteristics. The primary outcome was all-cause mortality. In patients whose outcome could not be confirmed, the follow-up was ended on their last visit in June 2017.

\section{Ethical approval}

The ethics committee of the Showa University Fujigaoka Hospital approved the present study (F2018C13).

\section{Data collection}

Data were retrospectively collected from the participants' electronic medical records at admission. This included their age, sex, systolic blood pressure (SBP), diastolic blood pressure (DBP), comorbidities, etiology, clinical scenario (CS) class, left ventricular ejection fraction (LVEF), number of medications, number of PIMHF, and medication use. Information on medications at admissions was retrieved from the comprehensive list compiled by pharmacists. Polypharmacy was defined as the use of $\geq 6$ medications at the time of admission.

The PIMs and Consensus PIMHF list were both defined by STOPP criteria. The 11 items in the Consensus PIMHF list were as follows: NSAIDs, COX-2 inhibitors, corticosteroids, decongestants, non-dihydropyridine calcium channel blockers, thiazolidinediones, metformin, pregabalin, beta 2 -agonists, itraconazole, and medicinal formulations with high sodium content. In this study, we investigated NSAIDs and COX-2 inhibitors. Furthermore, we investigated PIMs $\left(\mathrm{H}_{2}\right.$ antagonists, PPIs, BZPs, and non-BZP hypnotics) with high frequency of prescription in elderly patients in Japan. Sulfonylureas (SUs), which are known to cause severe hypoglycemia in elderly patients, were also investigated. Laboratory data, including hemoglobin (Hb), albumin (Alb), blood urea nitrogen (BUN), and estimated glomerular filtration rate (eGFR), were also collected from medical records at admission.

\section{Statistical analysis}

Categorical and consecutive data regarding patient background are presented as number $(\%)$ and the mean \pm standard deviation (SD), respectively. The $\chi^{2}$ test was used for comparisons between groups, and the $t$-test was used for comparison of consecutive variables. All-cause mortality was estimated using Kaplan-Meier curves and the log-rank test was used to assess the significance of differences between the groups. Multiple Cox regression analysis including variables with $\mathrm{P}$ values $<$ 0.05 was performed to determine the predictors of HF patients' prognosis. Statistical analyses were performed using SPSS software version 23.0 (IBM, Armonk, NY, USA).

\section{Results}

\section{Descriptive}

A total of 193 elderly patients (median age 81 years; interquartile range (IQR) 65 - 99 years; 109 (56.5\%) were male) were included in the study; 30 patients aged $\geq 65$ years were admitted due to all-cause mortality. The baseline characteristics of the patients are presented in Table 1. The etiology of these patients was as follows: ischemic heart disease $(n=67,34.7 \%)$, hypertensive disease $(\mathrm{n}=29,15.0 \%)$, and valvular disease $(\mathrm{n}=$ $31,16.1 \%)$. The most common comorbidities were as follows: hypertension $(\mathrm{n}=156,80.8 \%)$, atrial fibrillation/flutter $(\mathrm{n}=$ $96,49.7 \%)$, and dyslipidemia $(\mathrm{n}=87,45.1 \%)$. CS 1 and 2 were 
Table 1. Patient Characteristics $(n=193)$

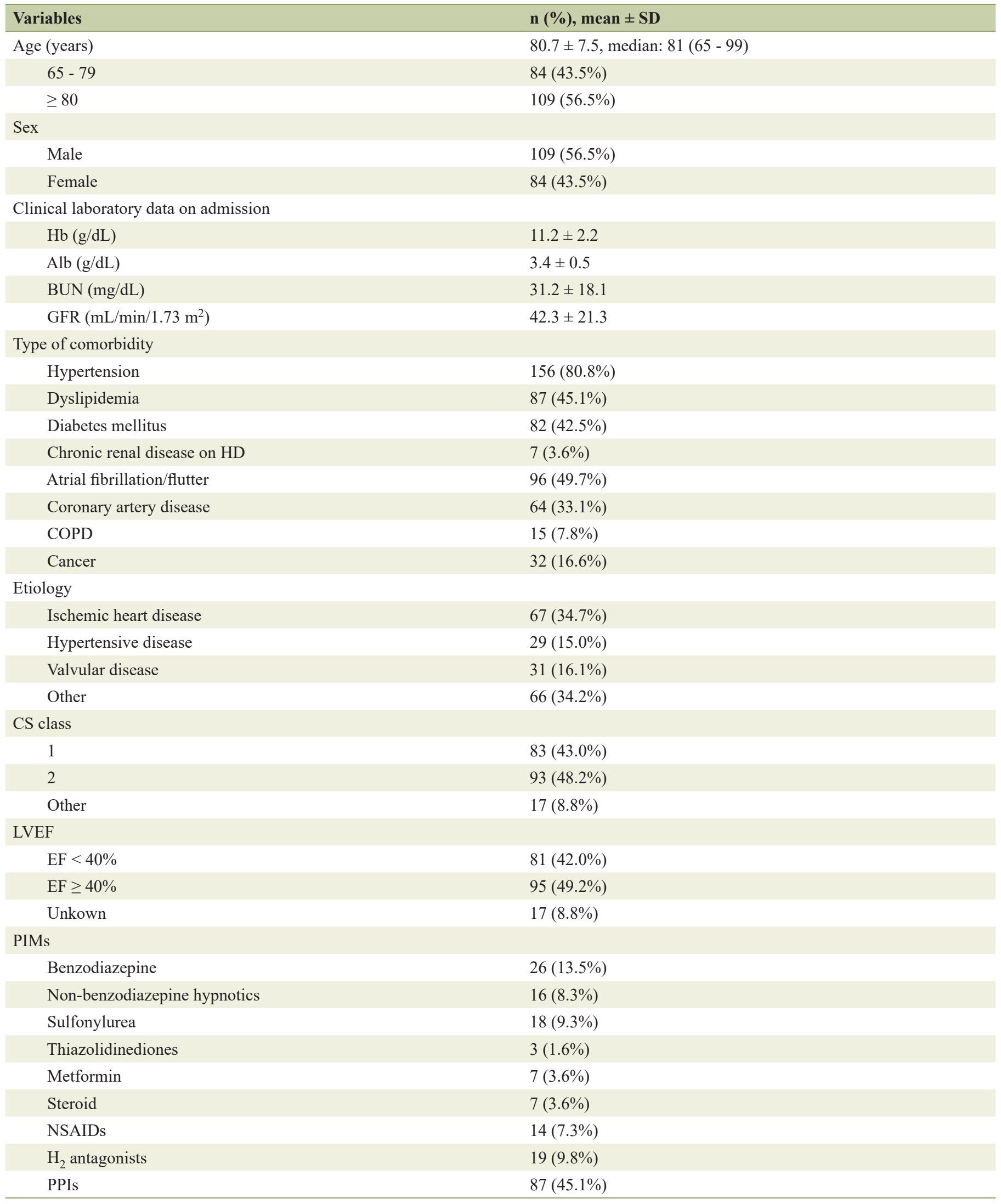


Table 1. Patient Characteristics $(n=193)$ - (continued)

\begin{tabular}{ll}
\hline Variables & $\mathbf{n}(\mathbf{\%})$, mean \pm SD \\
\hline Non-dihydropyridine calcium channel blockers & $10(5.2 \%)$ \\
Pregabalin & $2(1.4 \%)$ \\
Decongestants & 0 \\
Medicinal formulations with high sodium content & 0 \\
Beta $_{2}$-agonists & 0 \\
Itraconazole & 0 \\
\hline
\end{tabular}

SD: standard deviation; Hb: hemoglobin; Alb: albumin; BUN: blood urea nitrogen; GFR: glomerular filtration rate; HD: hemodialysis; COPD: chronic obstructive pulmonary disease; CS: clinical scenario; LVEF: left ventricular ejection fraction; PIM: potentially inappropriate medication; NSAIDs: nonsteroidal anti-inflammatory drugs; PPIs: proton pump inhibitors.

$83(43.0 \%)$ and $93(48.2 \%)$, respectively.

\section{Number of medications and common PIMs at admission}

Table 1 shows the number of medications prescribed at admission. Data show that $66.3 \%$ of the patients were receiving $\geq 6$ medications at admission with an average of $7.1 \pm 4.0$ medications per patient; $73.6 \%$ of the patients receiving at least one PIM at admission had an average of $1.1 \pm 0.9$ medications per patient. The most common PIMs used at admission were PPIs, BZPs, and $\mathrm{H}_{2}$ antagonists. These three categories comprised more than $60 \%$ of the total PIMs at admission.

\section{Relationship between various clinical factors including PIMs and all-cause mortality}

In the univariate analyses, Alb $(<3.5 \mathrm{~g} / \mathrm{dL})$, hypertension, chronic obstructive pulmonary disease (COPD), SBP $(<100$ $\mathrm{mm} \mathrm{Hg}$ ), number of medication ( $\geq 6)$, and NSAIDs were significantly associated with mortality (Table 2). Patients who did not take NSAIDs at admission showed a significantly lower all-cause mortality than those taking NSAIDs (2-year mortality $18.1 \%$ vs. $39.1 \%, \mathrm{P}=0.002$, Fig. 1 ). Patients who were taking $<6$ medicines at admission showed a significantly lower all-cause mortality than those taking $\geq 6$ medicines (2year mortality $10.0 \%$ vs. $25.0 \%, \mathrm{P}=0.045$, Fig. 2 ). However, there was no significant association between PIMs other than NSAIDs and mortality in ADHF patients.

COPD, SBP $<100 \mathrm{~mm} \mathrm{Hg}$, and NSAIDs remained significant in multivariate analysis (Table 3 ). The hazard ratios (HRs) for the presence to absence of the following variables were as follows: COPD, 3.855 (95\% confidence interval (CI), 1.417 - 10.489; P = 0.008); "SBP < $100 \mathrm{~mm} \mathrm{Hg"} \mathrm{to} \mathrm{"SBP} 100 \mathrm{~mm}$ Hg”, 5.341 (95\% CI, 2.204 - 12.942; P < 0.001); and NSAIDs, 4.646 (95\% CI, 1.725 - 12.510; $\mathrm{P}=0.002)$.

\section{Patient characteristics comparing non-NSAID and NSAID groups}

Patients taking NSAIDs prior to admission were prescribed a significantly larger number of medications at admission than those not taking NSAIDs (Table 4). Although patients taking NSAIDs did not have significantly increased SBP and DBP

Table 2. Univariate Analyses of Relationship Between Various Clinical Factors and Mortality (30 Events, $n=193$ )

\begin{tabular}{|c|c|c|}
\hline Variables & 2-year (\%) & Pvalue ${ }^{a}$ \\
\hline Age $\geq 80$ years & 20.6 & 0.378 \\
\hline Male sex & 19.2 & 0.711 \\
\hline Hypertension & 15.3 & $<0.001$ \\
\hline Diabetes mellitus & 18.5 & 0.660 \\
\hline Dyslipidemia & 18.8 & 0.229 \\
\hline Chronic renal disease on HD & 35.7 & 0.156 \\
\hline Atrial fibrillation/flutter & 20.2 & 0.672 \\
\hline Coronary artery disease & 28.1 & 0.420 \\
\hline COPD & 43.4 & 0.028 \\
\hline Cancer & 27.1 & 0.173 \\
\hline $\mathrm{SBP}<100 \mathrm{~mm} \mathrm{Hg}$ & 59.2 & $<0.001$ \\
\hline Number of medication $\geq 6$ at admission & 25.0 & 0.045 \\
\hline \multicolumn{3}{|l|}{ Common PIMs } \\
\hline Benzodiazepines & 13.6 & 0.970 \\
\hline Other than benzodiazepines & 15.4 & 0.867 \\
\hline Sulfonylurea & 13.4 & 0.482 \\
\hline Thiazolidinediones & 0.0 & 0.415 \\
\hline Metformin & 16.7 & 0.806 \\
\hline Steroid & 57.1 & 0.197 \\
\hline NSAIDs & 39.1 & 0.002 \\
\hline $\mathrm{H}_{2}$ antagonists & 33.9 & 0.252 \\
\hline PPIs & 28.8 & 0.080 \\
\hline $\mathrm{Hb}<12 \mathrm{~g} / \mathrm{dL}$ & 22.7 & 0.900 \\
\hline $\mathrm{Alb}<3.5 \mathrm{~g} / \mathrm{dL}$ & 26.6 & 0.011 \\
\hline $\mathrm{eGFR}<45 \mathrm{~mL} / \mathrm{min} / 1.73 \mathrm{~m}^{2}$ & 21.5 & 0.323 \\
\hline
\end{tabular}

aLog-rank test. HD: hemodialysis; COPD: chronic obstructive pulmonary disease; SBP: systolic blood pressure; PIM: potentially inappropriate medication; NSAIDs: non-steroidal anti-inflammatory drugs; PPIs: proton pump inhibitors; Hb: hemoglobin; Alb: albumin; eGFR: estimated glomerular filtration rate. 


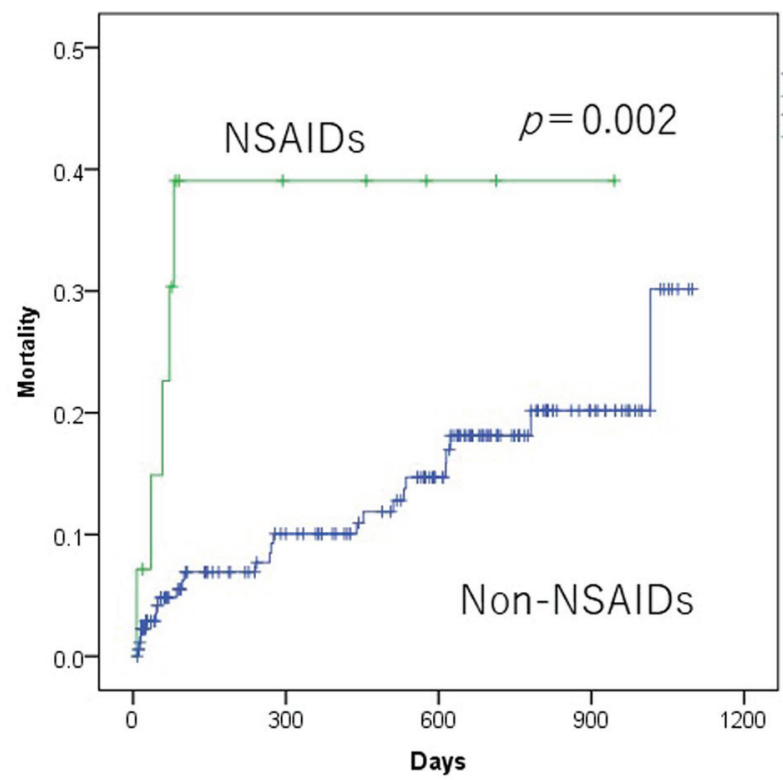

$\begin{array}{lccc}\text { Patients at risk } & & & \\ \text { Days } & 0 & 365 & 730 \\ \text { Number of Non-NSAIDs } & 178 & 107 & 47 \\ \text { Number of NSAIDs } & 13 & 4 & 1\end{array}$

Figure 1. Kaplan-Meier mortality curves according to non-steroidal anti-inflammatory drug use at admission.

levels at admission compared to patients without NSAIDs therapy, patients taking NSAIDs tended to have a higher SBP and DBP. Elderly patients taking NSAIDs also tended to have lower serum Alb levels.

\section{Discussion}

The present study shows that NSAIDs, SBP $<100 \mathrm{~mm} \mathrm{Hg}$, and COPD were independent prognostic factors to HF patients at admission. Patients who were prescribed NSAIDs, one of the PIMs, at the time of hospitalization showed 4.6 times poorer prognosis than those who were not prescribed. To the best of our knowledge, this is the first evidence demonstrating a relationship between PIMs and prognostic factor in HF patients.

Recently, several studies related to PIMs have reported that polypharmacy caused by aging has been regarded as a major problem $[7-9,11,15]$. In a study at a cardiology service center in USA, female sex, atrial fibrillation, cerebrovascular events, depression, history of fractures, and number of drugs were reported as factors related to PIMs, and that the proportion of PIM use is high in elderly patients with cardiovascular disease [8]. In Japan, there is a growing demand to increase and request the establishment of a basic law for the treatment of stroke and cardiovascular diseases for patients with HF. In this study, patients prescribed $\geq 6$ drugs had poor prognosis and NSAIDs, one of the PIMs, were associated with poor prognosis in HF patients. Feng et al reported that individuals with multimorbidity were more likely to have polypharmacy

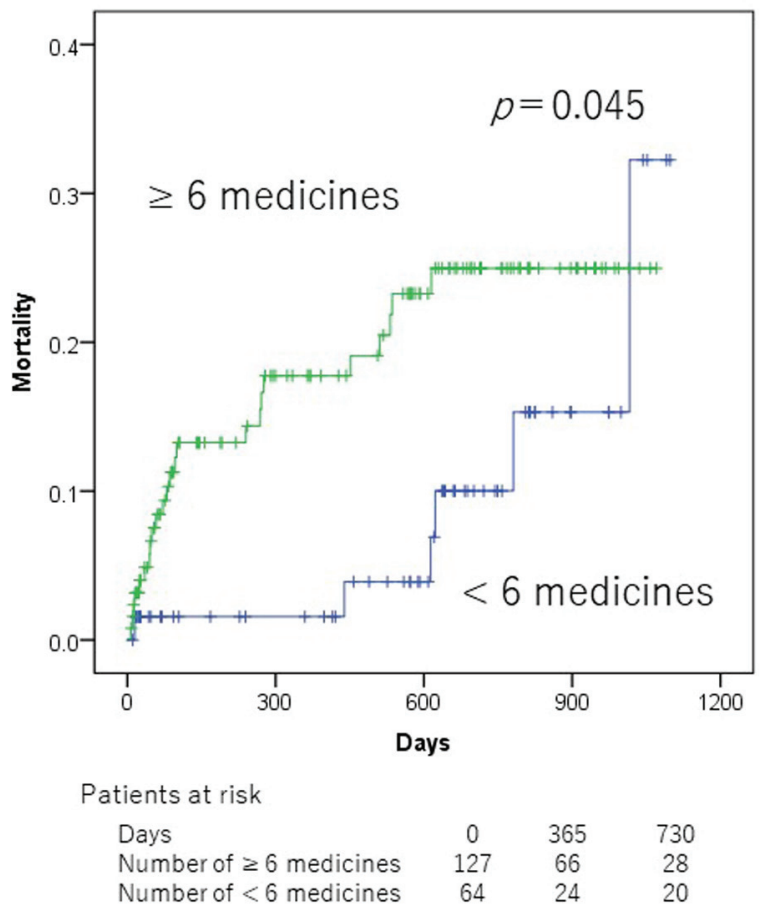

Figure 2. Kaplan-Meier mortality curves according to polypharmacy at admission.

than those without multimorbidity [16]. They also mentioned that patients with certain chronic conditions or combinations, such as a chronic kidney condition or HF accompanied with musculoskeletal conditions, may be at higher risk for polypharmacy. In our study, although we did not consider the number of comorbidities, patients prescribed $\geq 6$ drugs resulted to have poor prognosis. Therefore, multimorbidity itself may also be a metric of greater disease severity in those patients.

The therapeutic action of NSAIDs is mostly mediated by the inhibition of COX-2 [17]. The overall effects of this inhibition of the isoenzymes of prostaglandin $\mathrm{G} / \mathrm{H}$ synthase mediate the increase in peripheral systemic resistance and reduction in renal perfusion, glomerular filtration rate, and sodium excretion in susceptible individuals [18, 19]. These mechanisms could trigger the clinical manifestations of HF [20]. Because the level of prostaglandin inhibition mediated by NSAIDs increases with dose $[21,22]$, the risk of clinical HF could be expected to increase. In this study, we observed that patients on NSAIDs had a tendency to have higher values for SBP and DBP. As NSAIDs promote sodium and water retention, they eventually lead to hypertension, thus contributing to increased mortality. We found that patients with NSAIDs therapy were prescribed a significantly greater number of medications at admission than those without NSAIDs therapy. Elderly patients, taking NSAIDs, also tended to have lower serum Alb levels. On the other side, frailty, undernutrition, and infection may lead to HF. Therefore, patients taking NSAIDs might have multimorbidity, undernutrition, and frailty, related to spinal canal stenosis, knee joint disease, and post fracture status.

Arfe et al found an increased risk of hospital admission 
Table 3. Multivariate Analyses of the Relationship Between Various Clinical Factors and Mortality

\begin{tabular}{llll} 
Prognosis factors & $\boldsymbol{\beta}$ & HR (95\% CI) & P value $^{\mathbf{a}}$ \\
\hline NSAIDs, absent vs. present & 1.536 & $4.646(1.725-12.510)$ & 0.002 \\
SBP, $<100 \mathrm{~mm} \mathrm{Hg} \mathrm{vs.} \geq 100 \mathrm{~mm} \mathrm{Hg}$ & 1.675 & $5.341(2.204-12.942)$ & $<0.001$ \\
COPD, absent vs. present & 1.349 & $3.855(1.417-10.489)$ & 0.008 \\
\hline
\end{tabular}

${ }^{a}$ Cox regression for mortality. NSAIDs: non-steroidal anti-inflammatory drugs; SBP: systolic blood pressure; COPD: chronic obstructive pulmonary disease; HR: hazard ratio; $\mathrm{Cl}$ : confidence interval.

for HF in association with use of several traditional NSAIDs and two COX-2 inhibitors [23]. Although this study could not investigate the type of NSAIDs, it showed that NSAIDs as a group are associated with poor prognosis in HF patients. Therefore, when NSAIDs are prescribed in patients hospitalized for HF, it is crucial for doctors and pharmacists to fully evaluate the necessity of NSAIDs and to investigate whether their use is clearly needed.

On the contrary, diabetes mellitus (DM) medications such as thiazolidinediones, metformin, and sulphonylurea derivatives were not associated with all-cause mortality in HF patients. Thiazolidinediones are known to adversely cause sodium and water retention which could lead to an increased risk of worsening $\mathrm{HF}$ and hospitalization. Hence, their use is not recommended in patients with HF [24, 25]. Metformin is contraindicated in patients with severe renal or hepatic impairment, because of the risk of lactic acidosis. In our study, among the patients taking metformin, three patients were found to have eGFR less than $45 \mathrm{~mL} / \mathrm{min} / 1.73 \mathrm{~m}^{2}$. Sulphonylurea derivatives have also been associated with an increased risk of worsening $\mathrm{HF}$ and should be used with caution. A consensus report by the American Diabetes Association (ADA) and the European Association for the Study of Diabetes (EASD) [26] advises that for patients with atherosclerotic cardiovascular disease, the first line metformin should be followed by sodium-glucose cotransporter 2 (SGLT2) inhibitors and glucagon-like peptide 1 (GLP-1) receptor agonists. Because a cardiologist was highly aware of a PIM, there were several cases where it was changed to SGLT2 inhibitor or GLP-1 receptor agonist at hospitalization. This suggests that there was no relationship between the DM medicine being investigated and the prognosis of HF.
Lee et al reported that BZPs have been shown to increase the risk of falls in HF patients [27]. Even in the guidelines in Japan [14], BZP-based hypnotic agents in the elderly are recommended to be used only for a short period with minimal use as it worsens adverse events such as delirium, falls, and fractures. In the present study, $12.8 \%$ of HF patients prescribed BZP-based hypnotic agents were not associated with all-cause mortality. Since this study did not consider the onset of action, such detailed examination would be necessary for future studies.

Yoshihisa et al reported that PPIs may be associated with improving outcomes in HF patients [28]. In addition, several studies have investigated the association between PPI use and fracture, bone loss, inhibition of gastric acid secretion, and clostridium decile infection [29]. From 2012 to 2015, the American Geriatrics Society has updated the Beers Criteria to include PPI in the list of PIMs [12]. A high proportion of patients with a history of ischemic heart disease are typically prescribed antiplatelet drugs. A similar observation has been reported in patients with atrial fibrillation who are taking anticoagulant drugs. It was observed that the prescription rate of $\mathrm{H}_{2}$ antagonists and PPIs was high in these patients [7]. The same observation was also found in the present study. This highlights the key role of clinical pharmacists to evaluate whether drug treatment with PPI is necessary.

Two factors, mainly COPD and SBP $<100 \mathrm{~mm} \mathrm{Hg}$, were also prognostic factors for $\mathrm{HF}$ in this study. The Acute Decompensated Heart Failure Syndromes (ATTEND) registry of acute HF epidemiologic research shows that HF patients with lower SBP at admission in the elderly have poorer prognosis [30]. In this study, HF patient with SBP $<100 \mathrm{~mm} \mathrm{Hg}$ at hospitalization had poor prognosis and similar results. In some

Table 4. Patient Characteristics Comparing Non-NSAID and NSAID Groups

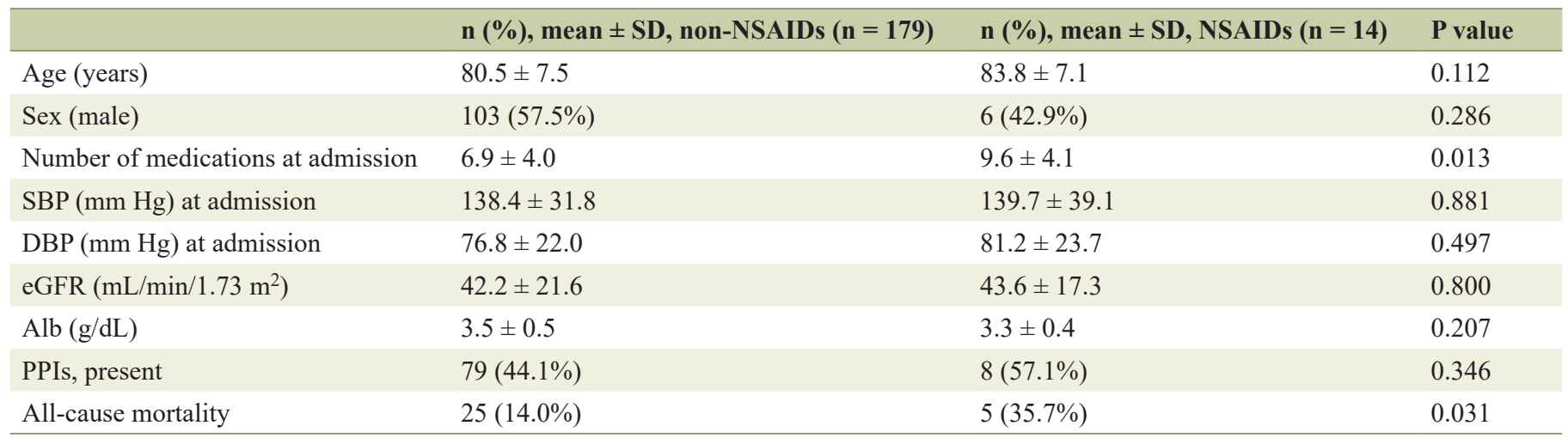

NSAIDs: non-steroidal anti-inflammatory drugs; SD: standard deviation; SBP: systolic blood pressure; DBP: diastolic blood pressure; eGFR: estimated glomerular filtration rate; Alb: albumin; PPIs: proton pump inhibitors. 
studies, COPD has been frequently observed as a comorbidity in HF patients, and it is associated with the all-cause mortality [31-33]. Similar results were observed in this study. This suggests that HF patients with $\mathrm{SBP}<100 \mathrm{~mm} \mathrm{Hg}$ at hospitalization and COPD are considered to have poor prognosis. Therefore, it is necessary to determine the treatment policy and examine the patient environment after discharge.

\section{Limitation}

The present study has some limitations. First, our study was conducted at a single institution, and thus, the sample size was relatively small. Second, we could not exclude the possibility of residual confounders as the result of missing data, such as the use of over-the-counter $\mathrm{H}_{2}$ antagonists and meals high in sodium content. Third, the average follow-up period was approximately 12 months in our study, which may be insufficient to fully evaluate the long-term prognosis of all-cause mortality. Fourth, patients may have been readmitted, which would have led to an underestimation of adherence. In addition, we have not assessed the living environment and dietary condition of patients which may have led to worsening of HF. Fifth, we could not keep track of whether there were medications prescribed from other medical institutions. We did not investigate the medicines prescribed at discharge, but other than one patient who continued to take NSAIDs after discharge or died during hospitalization.

\section{Conclusions}

We demonstrated that NSAIDs were independently associated with all-cause mortality in ADHF patients at admission. The medical staff should attempt to stop unnecessary medications that are prone to inappropriate prescribing. In particular, NSAIDs should be carefully assessed and monitored.

\section{Acknowledgments}

None to declare.

\section{Financial Disclosure}

None to declare.

\section{Conflict of Interest}

TM received personal fees from Heart Flow Inc. The other authors have no conflict of interest.

\section{Informed Consent}

Not applicable.

\section{Author Contributions}

T. Sunaga, AY, TM, ME and TW contributed to conceptualization; T. Sunaga, AY and SW contributed to methodology; S. Nakamura, NM, SW, MT, S. Nagumo, A. Nogi, HM and TM contributed to investigation; T. Sunaga, AY and SW contributed to analysis; T. Sunaga contributed to resources, writing and original draft preparation; T. Sunaga, ME, HS, A. Nakamura, TW and T. Sunaga were involved in writing and editing; HS, A. Nakamura and T. Sasaki contributed to supervision; T. Sunaga was responsible for project administration. The final version of the paper was seen and approved by all authors.

\section{Data Availability}

The authors declare that data supporting the findings of this study are available within the article.

\section{References}

1. Shimokawa H, Miura M, Nochioka K, Sakata Y. Heart failure as a general pandemic in Asia. Eur J Heart Fail. 2015;17(9):884-892.

2. Kaneko H, Suzuki S, Goto M, Arita T, Yuzawa Y, Yagi $\mathrm{N}$, Murata N, et al. Incidence and predictors of rehospitalization of acute heart failure patients. Int Heart J. 2015;56(2):219-225.

3. Wolff JL, Starfield B, Anderson G. Prevalence, expenditures, and complications of multiple chronic conditions in the elderly. Arch Intern Med. 2002;162(20):2269-2276.

4. Kaufman DW, Kelly JP, Rosenberg L, Anderson TE, Mitchell AA. Recent patterns of medication use in the ambulatory adult population of the United States: the Slone survey. JAMA. 2002;287(3):337-344.

5. Steinman MA, Landefeld CS, Rosenthal GE, Berthenthal D, Sen S, Kaboli PJ. Polypharmacy and prescribing quality in older people. J Am Geriatr Soc. 2006;54(10):15161523.

6. Tommelein E, Mehuys E, Petrovic M, Somers A, Colin $\mathrm{P}$, Boussery K. Potentially inappropriate prescribing in community-dwelling older people across Europe: a systematic literature review. Eur J Clin Pharmacol. 2015;71(12):1415-1427.

7. Komagamine J. Prevalence of potentially inappropriate medications at admission and discharge among hospitalised elderly patients with acute medical illness at a single centre in Japan: a retrospective cross-sectional study. BMJ Open. 2018;8(7):e021152.

8. Sheikh-Taha M, Dimassi H. Potentially inappropriate home medications among older patients with cardiovascular disease admitted to a cardiology service in USA. BMC Cardiovasc Disord. 2017;17(1):189.

9. Ledwidge M, Travers B, Ryder M, Ryan E, McDonald K. Specialist care of heart failure improves appropriate pharmacotherapy at the expense of greater polypharmacy and drug-interactions. Eur J Heart Fail. 2004;6(2):235-243. 
10. McMurray JJ, Adamopoulos S, Anker SD, Auricchio A, Bohm M, Dickstein K, Falk V, et al. ESC guidelines for the diagnosis and treatment of acute and chronic heart failure 2012: the task force for the diagnosis and treatment of acute and chronic heart failure 2012 of the European Society of Cardiology. Developed in collaboration with the Heart Failure Association (HFA) of the ESC. Eur J Heart Fail. 2012;14(8):803-869.

11. Wong CY, Chaudhry SI, Desai MM, Krumholz HM. Trends in comorbidity, disability, and polypharmacy in heart failure. Am J Med. 2011;124(2):136-143.

12. By the American Geriatrics Society Beers Criteria Update Expert P. American Geriatrics Society 2015 Updated Beers Criteria for Potentially Inappropriate Medication Use in Older Adults. J Am Geriatr Soc. 2015;63(11):22272246.

13. O'Mahony D, O'Sullivan D, Byrne S, O'Connor MN, Ryan C, Gallagher P. STOPP/START criteria for potentially inappropriate prescribing in older people: version 2 . Age Ageing. 2015;44(2):213-218.

14. https://www.jpn-geriat-soc.or.jp/info/topics/ pdf/20170808_01.pdf.

15. Bermingham $\bar{M}$, Ryder M, Travers B, Edwards N, Lalor L, Kelly D, Gallagher J, et al. The St Vincent's potentially inappropriate medicines study: development of a diseasespecific consensus list and its evaluation in ambulatory heart failure care. Eur J Heart Fail. 2014;16(8):915-922.

16. Feng X, Tan X, Riley B, Zheng T, Bias T, Sambamoorthi U. Polypharmacy and multimorbidity among medicaid enrollees: a multistate analysis. Popul Health Manag. 2018;21(2):123-129.

17. FitzGerald GA, Patrono C. The coxibs, selective inhibitors of cyclooxygenase-2. N Engl J Med. 2001;345(6):433442.

18. Patrono $\mathrm{C}$, Baigent $\mathrm{C}$. Nonsteroidal anti-inflammatory drugs and the heart. Circulation. 2014;129(8):907-916.

19. Bleumink GS, Feenstra J, Sturkenboom MC, Stricker BH. Nonsteroidal anti-inflammatory drugs and heart failure. Drugs. 2003;63(6):525-534.

20. Huerta C, Varas-Lorenzo C, Castellsague J, Garcia Rodriguez LA. Non-steroidal anti-inflammatory drugs and risk of first hospital admission for heart failure in the general population. Heart. 2006;92(11):1610-1615.

21. Garcia Rodriguez LA, Hernandez-Diaz S. Nonsteroidal antiinflammatory drugs as a trigger of clinical heart failure. Epidemiology. 2003;14(2):240-246.

22. Back M, Yin L, Ingelsson E. Cyclooxygenase-2 inhibitors and cardiovascular risk in a nation-wide cohort study after the withdrawal of rofecoxib. Eur Heart J. 2012;33(15):1928-1933.

23. Arfe A, Scotti L, Varas-Lorenzo C, Nicotra F, Zambon A, Kollhorst B, Schink T, et al. Non-steroidal anti-inflammatory drugs and risk of heart failure in four European countries: nested case-control study. BMJ. 2016;354:i4857.

24. Hernandez AV, Usmani A, Rajamanickam A, Moheet A. Thiazolidinediones and risk of heart failure in patients with or at high risk of type 2 diabetes mellitus: a meta-analysis and meta-regression analysis of placebocontrolled randomized clinical trials. Am J Cardiovasc Drugs. 2011;11(2):115-128.

25. Komajda M, McMurray JJ, Beck-Nielsen H, Gomis R, Hanefeld M, Pocock SJ, Curtis PS, et al. Heart failure events with rosiglitazone in type 2 diabetes: data from the RECORD clinical trial. Eur Heart J. 2010;31(7):824-831.

26. Davies MJ, D'Alessio DA, Fradkin J, Kernan WN, Mathieu C, Mingrone G, Rossing P, et al. Management of Hyperglycemia in Type 2 Diabetes, 2018. A consensus report by the American Diabetes Association (ADA) and the European Association for the Study of Diabetes (EASD). Diabetes Care. 2018;41(12):2669-2701.

27. Lee K, Pressler SJ, Titler M. Falls in patients with heart failure: a systematic review. J Cardiovasc Nurs. 2016;31(6):555-561.

28. Yoshihisa A, Takiguchi M, Kanno Y, Sato A, Yokokawa T, Miura S, Abe S, et al. Associations of acid suppressive therapy with cardiac mortality in heart failure patients. J Am Heart Assoc. 2017;6(5).

29. Katz PO, Gerson LB, Vela MF. Guidelines for the diagnosis and management of gastroesophageal reflux disease. Am J Gastroenterol. 2013;108(3):308-328; quiz 329.

30. Kajimoto K, Sato N, Takano T, investigators of the Acute Decompensated Heart Failure Syndromes r. Association of age and baseline systolic blood pressure with outcomes in patients hospitalized for acute heart failure syndromes. Int J Cardiol. 2015;191:100-106.

31. Le Jemtel TH, Padeletti M, Jelic S. Diagnostic and therapeutic challenges in patients with coexistent chronic obstructive pulmonary disease and chronic heart failure. J Am Coll Cardiol. 2007;49(2):171-180.

32. Niewoehner DE. Clinical practice. Outpatient management of severe COPD. N Engl J Med. 2010;362(15):14071416.

33. Khawaja FJ, Shah ND, Lennon RJ, Slusser JP, Alkatib AA, Rihal CS, Gersh BJ, et al. Factors associated with 30-day readmission rates after percutaneous coronary intervention. Arch Intern Med. 2012;172(2):112-117. 